\title{
BMJ Open Safety and efficacy of antenatal milk expressing for women with diabetes in pregnancy: protocol for a randomised controlled trial
}

\author{
Della A Forster, ${ }^{1,2}$ Susan Jacobs, ${ }^{2,3,4}$ Lisa H Amir, ${ }^{1}$ Peter Davis, ${ }^{2}$ Susan P Walker, ${ }^{4,5}$ \\ Kerri McEgan, ${ }^{5}$ Gillian Opie, ${ }^{4,5}$ Susan M Donath, ${ }^{6}$ Anita M Moorhead, ${ }^{1,2}$ \\ Rachael Ford, ${ }^{1,2}$ Catharine McNamara, ${ }^{5}$ Amanda Aylward, ${ }^{2}$ Lisa Gold ${ }^{7}$
}

To cite: Forster DA, Jacobs S, Amir LH, et al. Safety and efficacy of antenatal milk expressing for women with diabetes in pregnancy: protocol for a randomised controlled trial. BMJ Open 2014;4:e006571. doi:10.1136/bmjopen-2014006571

- Prepublication history and additional material is available. To view please visit the journal (http://dx.doi.org/ 10.1136/bmjopen-2014006571).

Received 12 September 2014 Revised 2 October 2014 Accepted 6 October 2014

CrossMark

For numbered affiliations see end of article.

Correspondence to Professor Della Forster; d.forster@latrobe.edu.au

\section{ABSTRACT}

Introduction: Many maternity providers recommend that women with diabetes in pregnancy express and store breast milk in late pregnancy so breast milk is available after birth, given (1) infants of these women are at increased risk of hypoglycaemia in the first $24 \mathrm{~h}$ of life; and (2) the delay in lactogenesis II compared with women without diabetes that increases their infant's risk of receiving infant formula. The Diabetes and Antenatal Milk Expressing (DAME) trial will establish whether advising women with diabetes in pregnancy (pre-existing or gestational) to express breast milk from 36 weeks gestation increases the proportion of infants who require admission to special or neonatal intensive care units (SCN/NICU) compared with infants of women receiving standard care. Secondary outcomes include birth gestation, breastfeeding outcomes and economic impact.

Methods and analysis: Women will be recruited from 34 weeks gestation to a multicentre, two arm, unblinded randomised controlled trial. The intervention starts at 36 weeks. Randomisation will be stratified by site, parity and diabetes type. Women allocated to the intervention will be taught expressing and encouraged to hand express twice daily for 10 min and keep an expressing diary. The sample size of 658 (329 per group) will detect a $10 \%$ difference in proportion of babies admitted to SCN/NICU (85\% power, $\alpha 0.05)$. Data are collected at recruitment (structured questionnaire), after birth (abstracted from medical record blinded to group), and 2 and 12 weeks postpartum (telephone interview). Data analysis: the intervention group will be compared with the standard care group by intention to treat analysis, and the primary outcome compared using $\chi^{2}$ and ORs. Ethics and dissemination: Research ethics approval will be obtained from participating sites. Results will be published in peer-reviewed journals and presented to clinicians, policymakers and study participants.

Trial registration number: Australian Controlled Trials Register ACTRN12611000217909.
Strengths and limitations of this study

- This will be the first study to provide rigorous evidence regarding the practice of antenatal expression of colostrum in late pregnancy for women with diabetes in pregnancy.

- It will explore the safety and efficacy for mother, fetus and infant.

- The conclusions about antenatal expressing will be restricted to women with diabetes in pregnancy, but if this practice is found to be safe and effective for this high-risk group, the conclusions are likely to be applicable to other women as well.

\section{BACKGROUND}

Diabetes is the second highest contributor to loss of health in Australia. ${ }^{1}$ Type 2 diabetes and gestational diabetes (GDM) are increasing globally. ${ }^{2}$ GDM occurs on average in $7 \%$ of pregnancies (range 1-14\% depending on the population characteristics and diagnostic tests used), ${ }^{2}$ and is the strongest single population predictor of type 2 diabetes. ${ }^{3}$ An additional $1 \%$ of females aged below 44 years have pre-existing diabetes (type 1 or 2 ), ${ }^{4}$ with type 2 diabetes increasing in women of childbearing age. ${ }^{5}$ Since 2009, women found to have glucose intolerance at the first pregnancy visit are diagnosed as having type 2 diabetes (not GDM). ${ }^{2}$ In Victoria in 2008, $6.1 \%$ of all women giving birth had diabetes in pregnancy (personal communication, A Cooper, Perinatal Data Collection Unit). At the planned original study sites, the Royal Women's Hospital (RWH) and Mercy Hospital for Women (MHW), 7.7\% (495/ $6443)$ and $10.3 \%(690 / 5748)$ of women, respectively, giving birth in 2009 had diabetes in pregnancy.

Pregnancies affected by diabetes have increased risk of perinatal complications, 
including increased perinatal mortality for infants of women with type 1 diabetes. $^{6-8}$ In GDM, poorer glycaemic control is also associated with adverse infant outcomes. $^{9}$

Infants of women with diabetes in pregnancy are at increased risk of hypoglycaemia (secondary to hyperinsulinism) and other morbidities in the early neonatal period (eg, macrosomia, respiratory distress syndrome, prematurity, congenital anomalies, polycythaemia, jaundice). ${ }^{510}$ They are more likely to themselves develop diabetes, and have an increased risk of obesity later in life. ${ }^{5}$

A strategy to decrease the risk of these infants developing diabetes or impaired glucose tolerance later in life would be one way of interrupting the cycle of diabetes. One such strategy is to increase the rate of exclusive breastfeeding from birth in these infants, given that early exposure to cow's milk protein increases the incidence of both type 1 (juvenile onset) and later onset type 2 diabetes. ${ }^{11-13}$ However, infants of women with diabetes are at high risk of not being exclusively breastfed for a number of reasons. Studies have found generally that women with diabetes are less likely to breastfeed than other women, and likely to breastfeed for a shorter duration, ${ }^{14-17}$ although one study has found that where breastfeeding is encouraged and supported, women with diabetes can be just as successful as women without diabetes. $^{18}$

Lactogenesis II, the onset of copious milk production, usually occurs $30-40 \mathrm{~h}$ after birth. ${ }^{19}$ Women with diabetes experience up to a $24 \mathrm{~h}$ delay in lactogenesis II compared with women without diabetes, ${ }^{20-24}$ as do women with glucose intolerance, increasing their infants' risk of receiving infant formula. ${ }^{25}$ Women with diabetes are also at risk of other comorbidities, most commonly obesity. ${ }^{26}{ }^{27}$ In turn, obese women ${ }^{28}$ and obese women with diabetes ${ }^{29}$ are less likely to successfully breastfeed. Separation of mother and infant following caesarean birth (also more likely in women with diabetes in pregnancy ${ }^{27}{ }^{30}$ ) and/or admission to special and neonatal intensive care units (SCN/NICU) further decreases the likelihood of establishing breastfeeding. ${ }^{31} 32$

Since infants of women with diabetes are at increased risk of hypoglycaemia, ${ }^{10}$ they require blood glucose monitoring and are often admitted to the SCN. If infants are hypoglycaemic and their mother is unable to provide a sufficient volume of expressed breast milk in addition to breastfeeding, they may receive supplementation with infant formula or intravenous glucose. This has led to a practice whereby some women with diabetes in pregnancy are being advised to express breast milk before their infant's birth.

\section{Current practice}

Increasing numbers of maternity providers are encouraging pregnant women with diabetes to express and store breast milk (colostrum) in the last weeks before their expected delivery date, ${ }^{33-38}$ so that breast milk is available in the postpartum period, thereby possibly avoiding infant formula if neonatal hypoglycaemia needs management with supplementary formula feeding. Some organisations have implemented guidelines for antenatal expressing of colostrum, ${ }^{39}{ }^{40}$ and in a recent book for consumers, there is a section entitled "Getting a head start: expressing milk before your baby is born" (ref.41, p.57). This practice presupposes that establishing a supply of colostrum prebirth might ameliorate: (1) the number of infants of women with diabetes who receive infant formula or intravenous glucose if supplementary feeding is required to treat neonatal hypoglycaemia (stored colostrum could be used); (2) the delay in lactogenesis II in women with diabetes and (3) the number of infants of women with diabetes admitted to the SCN. These recommendations for practice have not been investigated and are therefore theoretical, and there is very limited evidence regarding the safety or efficacy of encouraging antenatal expressing of breast milk.

\section{The evidence}

A retrospective cohort study from the UK included 94 women, and found that infants of women with diabetes who expressed were born 1 week earlier on average (37.1 weeks (SD 2.6), compared with 38.2 weeks (SD 2.2), $\mathrm{p}=0.06$ ), and were more likely to be admitted to the SCN. ${ }^{42}$ We conducted a pilot study in 2007 that also provided some evidence, and provided the baseline data to undertake the proposed randomised controlled trial (RCT) ${ }^{43}$ We recruited 43 women with diabetes in pregnancy who agreed to undertake antenatal expressing (35\% of those eligible agreed to participate when invited) and we conducted a concurrent audit of clinical outcomes in a group of infants of similar women (with diabetes in pregnancy) to provide 'control' data. Women in the expressing pilot completed a demographic questionnaire, were then taught how to express colostrum and encouraged to do so for $10 \mathrm{~min}$ twice daily from 36 weeks gestation. They were advised on the safe storage of colostrum, which they froze for their baby's use after birth. Women kept a diary documenting their expressing and completed telephone interviews at 6 and 12 weeks postpartum. We found that $30 \%$ of infants in the pilot were admitted to SCN compared with $17 \%$ of 'control' infants, and that reasons for admission to SCN were similar in the two groups. ${ }^{43}$ Intravenous glucose use was $14 \%$ for pilot infants and $8 \%$ for control infants $(\mathrm{RR}=1.77 ; 95 \% \mathrm{CI} 0.63$ to 4.96$)$. There was no evidence of any fetal compromise based on cardiotocographs (CTGs) undertaken after the first expressing episode. Women recorded blood sugar levels (BSLs) following their first three expressing episodes. Although the median BSLs were normal and suggested no evidence of hypoglycaemia at a group level, $10 \%(2 / 20)$ of women had a BSL $<3.5 \mathrm{mmol} / \mathrm{L}$ after the first expressing episode, and seven women $(27 \% ; 7 / 29)$ reported they experienced tightenings or Braxton Hicks contractions as a result of antenatal expressing, and one ceased the intervention for this reason. 
The amount of colostrum women obtained varied according to number of expressions, length of time between onset of expressing and birth and the time spent expressing, with a median of 14 days expressing and $40 \mathrm{~mL}$ (range $5-310 \mathrm{~mL}$ ) obtained. Although some women found expressing difficult $(31 \%)$, the intervention was positively received overall, and $95 \%(38 / 40)$ of women would express antenatally again if the practice was proven beneficial. More infants in the pilot received exclusive breast milk during their postpartum hospital stay $(37 \%)$ compared with the 'control' group (27\%; $\mathrm{RR}=1.38 ; 95 \%$ CI 0.82 to 2.31 ).

In January 2010, we conducted a telephone survey of 48 tertiary and large metropolitan and regional maternity hospitals across Australia providing care for 109465 births per year and found that 30 of these services (63\%; 65478 births) recommend antenatal milk expressing. ${ }^{44}$ Of these, $21(70 \%)$ recommended antenatal expressing primarily to women with diabetes in pregnancy (although the practice is also being recommended to women with other high-risk pregnancies), and $11(37 \%)$ had a policy or guideline for antenatal milk expressing. Across the 11 services with a policy or guideline for antenatal milk expressing, the mean gestation recommended for start of expressing was 36 weeks (range 30-37 weeks). Of the 18 services (38\%; 43987 births) who did not recommend this practice, 5 (28\%) discontinued antenatal expressing based on the recommendations of our pilot observational study, ${ }^{43}$ and await evidence to inform this practice. ${ }^{44}$

A recent Cochrane review identified no RCTs that investigated the practice of expressing and storing breast milk during pregnancy, and concluded that there is no high-level systematic evidence to inform the safety and efficacy of this practice. ${ }^{38}$ Rigorous evidence is urgently required to inform the clinical practice of antenatal expression of colostrum. ${ }^{44}$ This paper describes the protocol for an adequately powered RCT exploring the practice of advising women with diabetes in pregnancy to express breast milk from 36 weeks gestation.

\section{METHODS}

A multicentre, two arm, unblinded RCT design will be used to compare the practice of antenatal milk expressing with standard care, for women with pre-existing or GDM. In the original trial design we included only women with diabetes in pregnancy who required insulin, choosing this group because they are the women for whom antenatal expressing is most often suggested, yet are at the highest risk of perinatal complications, particularly if glycaemic control is poor. This inclusion criterion changed, as detailed below in the sample size section.

\section{Aims}

Primary aim

To establish whether the practice of antenatal expressing of colostrum from 36 weeks gestation, for women with diabetes in pregnancy, increases the proportion of infants who require admission to the SCN or NICU compared with the infants of similar women receiving standard care.

\section{Primary hypothesis}

Infants of women with diabetes in pregnancy who start antenatal expressing of colostrum from 36 weeks gestation will be more likely to be admitted to the SCN or NICU during the primary hospitalisation after birth compared with the infants of women with diabetes in pregnancy receiving standard care.

\section{Secondary aims}

To determine whether antenatal expressing of colostrum from 36 weeks gestation for women with diabetes in pregnancy, compared with similar women receiving standard care:

A. Increases the proportion of infants receiving exclusive breast milk at 3 months of age (ie, is effective in promoting exclusive breastfeeding);

B. Decreases the mean gestation at birth (ie, is harmful);

C. Increases the proportion of infants receiving exclusive breast milk during initial hospital stay (ie, is effective).

We will also:

D. Test the cost and cost-effectiveness of this intervention compared with standard care;

E. Explore the views and experiences of women participating in this trial;

F. Collect data on other outcomes-for example, fetal well-being associated with expressing; volumes of antenatal colostrum obtained; time to onset of lactogenesis II (onset of copious milk production).

Table 1 summarises the potential harms and benefits of antenatal expressing of colostrum.

Table 1 Potential harms and benefits of antenatal breast milk expressing

\begin{tabular}{|c|c|}
\hline Potential harms & Potential benefits \\
\hline $\begin{array}{l}\text { Increased admissions to } \\
\text { SCN/NICU (shows } \\
\text { increased neonatal } \\
\text { morbidity and } \\
\text { mother-infant separation) }\end{array}$ & $\begin{array}{l}\text { Increased exclusive } \\
\text { breastfeeding during initial } \\
\text { hospital stay } \\
\text { Increased exclusive } \\
\text { breastfeeding at } 3 \text { months } \\
\text { postpartum } \\
\text { Increased duration of any } \\
\text { breastfeeding }\end{array}$ \\
\hline $\begin{array}{l}\text { Decreased mean duration } \\
\text { of pregnancy } \\
\text { Increased requirement for } \\
\text { intravenous glucose }\end{array}$ & $\begin{array}{l}\text { Improved maternal } \\
\text { satisfaction } \\
\text { Decreased risk of } \\
\text { subsequent diabetes for the } \\
\text { infant }\end{array}$ \\
\hline
\end{tabular}




\section{Study sample}

All eligible women booking for maternity care at the trial sites during the recruitment period will be offered study participation.

Inclusion criteria: Any woman

1. With pre-existing or GDM;

2. Between 34 and 36 weeks gestation (but not more than 37 weeks);

3. With a singleton pregnancy in a cephalic presentation;

4. Attending the study sites for pregnancy care as a public patient;

5. Planning to breastfeed and

6. Able to speak and read in English.

Exclusion criteria: Women with

1. Any history of antepartum haemorrhage, or placenta praevia (even in the absence of any antenatal bleeding);

2. An unknown or classical caesarean section scar or more than one lower segment caesarean section scar;

3. Any suspicion of fetal compromise including known or suspected intrauterine growth restriction, documented macrosomia (estimated fetal weight $\geq 95$ th centile) with abdominal circumference $>97$ th centile, polyhydramnios or any abnormal tests of fetal wellbeing (whether clinically, ultrasound or CTG based);

4. A known fetal anomaly;

5. Hypertension and proteinuria-if any concerns about fetal well-being;

6. A serious maternal mental health issue, other severe maternal obstetric/medical issue.

In some sites there are small proportions of infants who are automatically admitted to the SCN after birth, for example, infants of women who have type 1 or 2 diabetes, or women on higher doses of insulin. In these sites, given the primary outcome is SCN/NICU admission, the women in those groups will be ineligible for inclusion in the trial.

\section{Recruitment}

Eligible women will be identified and offered participation by a study midwife at 34-36 weeks gestation, to maximise the opportunity to recruit women. If the woman is interested she will provide written consent and complete a questionnaire regarding demographic details and breastfeeding intentions. Randomisation occurs at 36 weeks gestation, so recruitment and randomisation will often be a two-stage process. Prior to randomisation women will have a preliminary 20 min CTG, and if the CTG is assessed as reactive and without significant uterine activity, the woman will be randomised to one of the two trial arms. Women allocated to the intervention will be taught hand expressing at that time (detailed more fully below), with a CTG during this first $10 \mathrm{~min}$ expressing episode and for $20 \mathrm{~min}$ after. The schedule of participant enrolment, intervention and assessments is shown in figure 1 .

\section{Randomisation procedure}

Randomisation is stratified by site, first baby or not, and diabetes type (ie, pre-existing (type 1 or 2 ), gestational requiring insulin or gestational not requiring insulin). A computerised random number generator was used to select random permuted blocks with at least three different block sizes. A system designed and administered by the Clinical Epidemiology and Biostatistics Unit at Murdoch Childrens Research Institute is accessed by the internet to ascertain women's allocation.

\section{The intervention}

Women randomised to the intervention will receive all standard advice and care (guided by existing hospital protocols) as well as receiving instructions on the intervention. They will be taught how to hand express colostrum (see online supplementary file 1). Women will be encouraged to express twice daily for no more than 10 min until being admitted to hospital to give birth, unless any concerns arise which indicate that the intervention should cease (see below).

Women will be provided with written and verbal instructions on the safe storage and transportation of colostrum (see online supplementary file 1). Expressed colostrum will be labelled with the woman's hospital medical record number and kept in syringes in her home freezer. Women will be asked to bring the frozen colostrum in an 'esky' (cold storage box) when they are admitted for the birth. Women will be provided with all the equipment they require for this intervention: syringes (2 and $5 \mathrm{~mL}$ ), small eskies, ice pack and specimen bags, labels with medical record number and diaries to document each episode of expressing.

A dedicated freezer will be available at each trial site for storage of antenatally expressed frozen colostrum. Midwifery and neonatal staff will receive education about the trial and be informed as to where to store the frozen expressed breast milk.

\section{Ensuring maternal and fetal well-being Surveillance in hospital}

Women in the intervention group will undertake an expressing episode under CTG surveillance at the time they are taught expressing (immediately after randomisation). If a woman requires further CTGs during their pregnancy care she will be asked to do one of her expressing episodes during those CTGs, as the uterus may become more sensitive to the resultant oxytocin surge with advancing gestation. The protocol for subsequent CTGs and expressing will be identical to that of the initial expressing episode, that is, preliminary CTG, express for $10 \mathrm{~min}$, then continue the CTG for $20 \mathrm{~min}$ postexpression. The CTG must be reactive prior to starting expressing. Immediate discontinuation of expressing will occur if there are any signs of fetal compromise (fetal tachycardia, reduced variability, late decelerations), or if there is excessive uterine activity (either a hypertonic contraction (one lasting longer than $90 \mathrm{~s}$ ), or 
Figure 1 Schedule of enrolment, intervention and assessments for the DAME (Diabetes and Antenatal Expressing) trial.

\begin{tabular}{|c|c|c|c|c|c|c|c|}
\hline & Enrolment & Allocation & \multicolumn{4}{|c|}{ Post-allocation } & Close- \\
\hline TIMEPOINT & $\begin{array}{c}\text { 34-36 } \\
\text { weeks of } \\
\text { pregnancy }\end{array}$ & $\begin{array}{l}36 \text { weeks } \\
\text { of } \\
\text { pregnancy }\end{array}$ & $\begin{array}{l}\text { Allo } \\
\text { cati } \\
\text { on }\end{array}$ & $\begin{array}{c}\text { Admi } \\
\text { ssion } \\
\text { for } \\
\text { birth }\end{array}$ & $\begin{array}{c}1-2 \\
\text { weeks } \\
\text { postp } \\
\text { artum }\end{array}$ & $\begin{array}{c}12 \\
\text { weeks } \\
\text { postpar } \\
\text { tum }\end{array}$ & $t_{x}$ \\
\hline ENROLMENT: & & & & & & & \\
\hline Eligibility screen & $x$ & & & & & & \\
\hline Informed consent & $x$ & & & & & & \\
\hline CTG & $x$ & & & & & & \\
\hline Allocation & & $x$ & & & & & \\
\hline INTERVENTIONS: & & & & & & & \\
\hline $\begin{array}{r}\text { Hand expressing } \\
\text { twice/day for } 10 \\
\text { mins }\end{array}$ & & & & & & & \\
\hline ASSESSMENTS: & & & & & & & \\
\hline $\begin{array}{r}\text { Maternal } \\
\text { characteristics } \\
\text { (in person) }\end{array}$ & $X$ & & & & & & \\
\hline $\begin{array}{r}\text { Maternal } \\
\text { expressing diary } \\
\text { (intervention } \\
\text { group) }\end{array}$ & & & & & & & \\
\hline $\begin{array}{l}\text { Obstetric data } \\
\text { (abstracted from } \\
\text { medical record) }\end{array}$ & & & & & & & $X$ \\
\hline $\begin{array}{l}\text { Neonatal data } \\
\text { (abstracted from } \\
\text { medical record) }\end{array}$ & & & & & & & $X$ \\
\hline $\begin{array}{r}\text { Infant feeding and } \\
\text { onset of lactation } \\
\text { data (telephone) }\end{array}$ & & & & & $x$ & & \\
\hline $\begin{array}{r}\text { Infant feeding data } \\
\text { (telephone) }\end{array}$ & & & & & & $x$ & \\
\hline Economic data & & & & & & & $\mathrm{X}$ \\
\hline
\end{tabular}

CTG: cardiotocograph tachysystole ( $>5$ contractions per $10 \mathrm{~min}$ period)). Each participating centre will follow their existing protocol for management of fetal distress, including availability of acute tocolysis and on-site obstetric support.

\section{Surveillance at home}

Women will be advised of precautions related to expressing in the antenatal period and informed that if they have concerns regarding any of these issues they should telephone the study midwife, or the emergency department/birth suite of their relevant hospital after hours. Instructions will be given regarding the importance of:

1. Noting normal fetal activity prior to expressing.

2. Reporting any complications after expressing, such as excessive uterine activity, vaginal blood loss, decreased fetal movements or signs of hypoglycaemia.

3. Measuring BSLs after the first three episodes of expressing, to ensure that the expressing is not causing hypoglycaemia (which can occur with breastfeeding). ${ }^{45}$ A consultant endocrinologist will advise the research team on maternal diabetes care as needed, and assist with interpretation of relevant outcomes.

\section{Standard care}

All women with pre-existing or GDM are seen by a diabetes educator for management of their diabetes. Additionally, all women discuss breastfeeding with midwives during pregnancy, and at all sites women can ask to see a lactation consultant in the antenatal period if they wish. No site can participate in the trial if they recommend that women express colostrum in the antenatal period.

\section{Management of neonatal hypoglycaemia}

The intervention being tested is not aimed at preventing hypoglycaemia; however, in practice the aim of many clinicians who advise antenatal milk expressing for this group is to avoid the use of formula for supplementary feeding if/when neonatal hypoglycaemia occurs. Based on our pilot data, approximately half of the admissions to SCN or NICU will be for neonatal hypoglycaemia. ${ }^{43}$ We will measure the incidence and duration of neonatal hypoglycaemia; however, it is not expected that encouraging women to express prior to birth could of itself prevent neonatal hypoglycaemia. 
Existing guidelines for management of newborn infants at risk of hypoglycaemia, including infants of women with diabetes in pregnancy, will be followed. While there may be slight variations in guidelines by trial site, for the purposes of data analysis neonatal hypoglycaemia is defined as a true blood glucose (TBG) $<2.6 \mathrm{mmol} / \mathrm{L}$ as measured on a blood gas analyser (or portable point of care TBG analyser) or in the laboratory. Sites whose guidelines for management of newborn infants at risk of hypoglycaemia are too different from this are not eligible to participate in the trial, and site guidelines are reviewed by the trial neonatologist prior to a decision about any site's suitability for inclusion. Although bedside testing with a glucometer is possible (providing BSLs), this method is not accurate at low blood glucose levels, when a TBG is mandatory. Prompt, standardised and accurate blood glucose measurement of TBG is optimal, ${ }^{46}{ }^{47}$ and in the trial will eliminate measurement bias in either trial arm. A portable point of care TBG analyser will facilitate prompt, accurate TBG measurement at the bedside without separation of mother and baby-crucial in supporting early breastfeeding.

Stratification by site ensures that any slight variation in infant glucose management will not affect trial outcomes. Regardless of group assignment, mothers will breastfeed their infants, and express their milk postpartum if required. The first response to hypoglycaemia (as per existing clinical guidelines at the two primary trial sites, RWH and MHW) is a prescribed volume (30$60 \mathrm{~mL} / \mathrm{kg}$ /day of supplemental feed of expressed breast milk or infant formula, or glucose gel $(0.5 \mathrm{~mL} / \mathrm{kg}$ of $40 \%)$ ) massaged into buccal mucosa. Hypoglycaemia unresponsive to supplemental feeding triggers admission to SCN/NICU, more frequent, larger supplemental feeds, further glucose gel or intravenous glucose therapy, to achieve normal TBGs.

\section{Process evaluation}

Adherence to the study protocol and intervention fidelity will be monitored.

1. Measures of intervention exposure: Women in the intervention group will keep a diary of antenatal expressing, noting: date and time of day each expressing episode takes place, length of each expressing episode and volume of colostrum expressed. Women in the control group will be asked at the 1-2 week interview if they expressed antenatally, to check for any cross-over.

2. Monthly meetings with all project staff will include discussion of protocol adherence, measurement and documentation.

3. Intervention evaluation by participants: The telephone interview at 12 weeks postpartum will include questions regarding women's views and experiences of being in the trial.

\section{Sample size}

Given the aim of antenatal expressing of breast milk is to benefit infants of women with diabetes in pregnancy, we debated whether our primary outcome should test efficacy or safety. Because our pilot data suggested potential harm, we chose the primary hypothesis to test safety. We are also powered to test the listed secondary outcomes.

\section{Primary outcome}

As aforementioned, when we designed the trial originally, we included only women with diabetes in pregnancy who required insulin, and our sample size calculations were based on estimates of this group. With that population we needed an estimated sample size of 658 women (329 in each group) based on $80 \%$ power $(\alpha 0.05)$, and allowing $5 \%$ loss to follow-up. This allowed the detection of an increase in the proportion of infants of women (requiring insulin in pregnancy) who are admitted to SCN or NICU in the primary hospital admission following birth, from $20 \%$ in the control group to $30 \%$ in the intervention group. This estimate was derived from the pilot and audit data presented above.

\section{Secondary outcomes}

This sample size also allows detection of:

A. A difference in exclusive breastfeeding at 3 months of $12 \%$ ( $40 \%$ in the control group compared with $52 \%$ in the intervention group; assuming the infants in the intervention achieve the same rate as the statewide data and as per our pilot, ie, $52 \%$; requires $\mathrm{n}=254$ );

B. A difference in the mean duration of pregnancy (38 weeks in the control group compared with 37 weeks in the intervention group; requires $n=184$; means, SDs derived from Soltani study ${ }^{42}$ ) and

C. A difference in exclusive breastfeeding during initial hospital stay of $12 \%$ (25\% in the control group compared with $37 \%$ in the intervention group).

\section{Sample size revision May 2012}

Recruitment to the trial as per the original criteria started in July 2011. In May 2012, it was agreed to amend our inclusion criteria to include all women with diabetes in pregnancy. This was due to two factors (A) the slow recruitment rate due to smaller numbers of eligible women than anticipated, and (B) expert advice from outside the research group recommending that the external validity of the trial would be increased if the criteria were broadened to also include women with diabetes in pregnancy who did not require insulin.

The change of inclusion criteria meant that a baseline rate of admission to the SCN or NICU would be $17 \%$ rather than $20 \%$ as per our original sample size calculations (based on a review of all 2011 outcome data for women with diabetes in pregnancy at the RWH). We undertook various recalculations to address this. Regarding the sample size with the altered baseline: 
- To detect $10 \%$ absolute difference (as in the original calculation) we would need 289 per group-this would be a decrease in sample size-which none of the team considered optimal;

- To detect a $50 \%$ relative increase to $25.7 \%$ would need 379 per group, that is, 758 (100 more than current sample of 658)-again this is not ideal as the study is not funded for this and we did not use this difference in our original calculations;

- Or we could maintain current sample size (658) and describe the study power to detect a $10 \%$ absolute difference.

The final option was chosen, that is, we agreed to maintain the current sample size and assume increased power to detect $10 \%$ absolute difference. Allowing for $5 \%$ loss to follow-up, the original sample size of 658 would provide 625 women at primary outcome measurement. This would detect a change in primary outcome from $17 \%$ to $27 \%$ with a power of $85 \%$. All the original secondary outcome comparisons described above will be maintained given the sample size has not altered.

\section{Outcome variables}

Primary outcome

Proportion of infants admitted to SCN or NICU.

\section{Secondary outcomes}

A. Proportion of infants receiving exclusive breast milk at 3 months of age;

B. Gestational age at birth;

C. Proportion of infants receiving exclusive breast milk during initial hospital stay;

D. Cost of the intervention to hospitals and to women and cost-effectiveness against breastfeeding outcomes;

E. Women's views and experiences;

F. Fetal well-being associated with expressing (assessed by CTG at initial then subsequent opportunistic CTG episodes); antenatal expression episodes, timing and volumes collected (intervention group only); time to lactogenesis II.

\section{Potential explanatory variables}

G. Reasons for SCN/NICU admissions;

H. Hypoglycaemia treatments in maternity or SCN/ NICU including glucose gel, intravenous glucose, glucagon, hydrocortisone;

I. Length of time until three consecutive infant TBG levels $\geq 2.6 \mathrm{mmol} / \mathrm{L}$;

J. Maternal blood glucose levels following first three expressing episodes;

K. Maternal morbidity that could be related to expressing, for example, premature labour.

\section{Data collection}

Maternal and infant

Demographic data (including age, education, marital status, ethnic background, smoking) will be collected by questionnaire at recruitment, prior to randomisation.
Obstetric/neonatal medical outcomes will be abstracted from the medical record following the birth by researchers blinded to group allocation.

Other outcome data will be collected by telephone administered questionnaire at 1-2 and 12 weeks postpartum. Women in the intervention arm will complete a diary of their expressing activity.

\section{Economic evaluation}

Resource use data will be collected from the medical record following birth and from women's self-reported use of health care and other resources in the 12 weeks after the birth. Costs included in the economic evaluation are those relating to care provided by the hospital (including admission to SCN/NICU) and women's (infants') use of healthcare and other societal resources over the period of the evaluation. Measured resource use will be valued using existing unit cost estimates (eg, Diagnosis Related Groups cost weights for hospital admissions for mother and infant ${ }^{48}$ and Medicare fee schedules for any attendances at the women's local doctor) ${ }^{49}$ As the primary outcome measure is itself a resource use item, economic evaluation will be expressed as cost-effectiveness analysis against exclusive breastfeeding at 3 months.

Blinding: The nature of the trial necessitates non-blinding of participants assigned to the intervention group, so that staff know to look for expressed breast milk in the freezer if it is available and required, and at most sites both the intervention and control groups need to be non-blinded to ensure that point of care TBGs are performed rather than BSLs, as most sites do BSLs unless indicated (rather than the TBGs required by this trial). Staff at sites that routinely undertake TBGs for all infants of women with diabetes in pregnancy will be blinded to women in the control group. Abstraction of medical record data will be undertaken blinded to group allocation; data will be presented to the data monitoring committee for the interim analysis in unlabelled study groups; and the research team will remain blinded to group allocation at all stages prior to final data analysis.

\section{Data analysis}

Data will be collected to meet the CONSORT guidelines for reporting of randomised trials ${ }^{50}$ including data on eligible non-participants. The first stage of analysis will check the comparability of participants allocated to the two groups. The intervention group will be compared with the standard care group by intention to treat analysis. The primary outcome measure will be compared using $\chi^{2}$ tests and ORs. Comparison of means will be undertaken for continuous variables using $t$ tests where data are normally distributed or Mann-Whitney U tests will compare medians otherwise. Ranked or Likert-type scales will be analysed using cumulative ORs. Where there are differences in baseline characteristics of the women in the two groups which might be associated with outcomes, an additional multivariate analysis will be carried out. Duration of breastfeeding will be compared 
using Kaplan-Meier statistics. Content analysis will be used to summarise open-ended comments. ${ }^{51}$

Data and Safety Monitoring Committees: A Data Monitoring Committee including a statistician, a midwife and an obstetrician (experienced in conducting RCTs) will undertake an interim analysis after half the women have given birth. A Safety Committee including a neonatologist, a midwife and an obstetrician (all experienced clinicians and researchers) will review reports of any adverse events, for example, an excess of any of the predefined adverse events for women or infants, blinded to group allocation (see online supplementary file 2 for list of events). Terms of reference for each of these committees will guide frequency of reviews and the conditions under which either committee would advise the research team that enrolment to the trial should cease.

\section{DISCUSSION}

This will be the first study to provide rigorous evidence regarding the practice of antenatal expression of colostrum in late pregnancy for women with diabetes in pregnancy. It will explore the safety and efficacy for mother, fetus and infant.

There remains widespread national and international interest in the outcomes of this trial, and the controversy can be seen in a recent review which concluded antenatal expressing should be encouraged: "Although Forster et $a t^{43}$ have argued the teaching of [antenatal expressing] should cease until the practice is proven to be safe and effective... an ethical dilemma now exists... as to whether the benefits of early colostrum feedings in at-risk babies outweighs the unproven side effect of premature labour. ${ }^{52}$ In the absence of evidence, the clear benefits of early feedings of colostrum should outweigh the unsupported risks of ceasing [antenatal expressing] education." 53

\section{TRIAL STATUS}

Approval has been granted from the following Human Research Ethics Committees (reference number in brackets): RWH (11/07); MHW (11/06); La Trobe University (11-004); Monash Medical Centre (12181-B); Barwon Health (13/06); Peninsula Health (14/PH/21).

Recruitment started at RWH in June 2011, followed by MHW in July 2011. In May 2012, due to (A) the slow recruitment rate resulting from low numbers of eligible women, and (B) external expert advice to include women with diabetes in pregnancy who did not require insulin, thereby increasing the trial's external validity, it was agreed to amend our inclusion criteria to include all women with diabetes in pregnancy (not just those requiring insulin) and to identify and recruit further trial sites. Monash Medical Centre started in October 2012 and Barwon Health (Geelong Hospital) in April 2013. The last site, Frankston Hospital, had ethics approval from Peninsula Health in July 2014 and recruitment will start soon.
In the original protocol women who had a history of spontaneous preterm birth or who had threatened preterm birth in the current pregnancy were excluded, however following the Safety Committee review of the first 100 births, which found no evidence of contractions following expressing, and because no woman is randomised prior to 36 weeks gestation, this exclusion criterion was removed.

\section{Monitoring CTGs}

The CTGS for all of the first 100 women who have given birth (prerandomisation, during and postexpressing) have been assessed by investigator SPW, with no sign of fetal compromise or increase in contractions as a result of expressing.

\section{Substudies}

1. Onset of lactation: In order to determine if the onset of lactation is delayed in women with diabetes, we are recruiting a comparison group of 200 women without diabetes. Women are being recruited in the postnatal wards at the RWH in 2014, and followed by telephone at 1-2 weeks postpartum, using identical questions to the DAME interview.

2. Antenatal colostrum: We plan to conduct a biochemical analysis of some excess samples of antenatal colostrum that infants have not required.

Author affiliations

1Judith Lumley Centre (formerly Mother \& Child Health Research), La Trobe University, Melbourne, Victoria, Australia

${ }^{2}$ Royal Women's Hospital, Parkville, Victoria, Australia

${ }^{3}$ Clinical Sciences, Murdoch Childrens Research Institute, Parkville, Victoria, Australia

${ }^{4}$ Department of Obstetrics and Gynaecology, University of Melbourne, Carlton, Victoria, Australia

${ }^{5}$ Mercy Hospital for Women, Heidelberg, Victoria, Australia

${ }^{6}$ Clinical Epidemiology and Biostatistics Unit, Murdoch Childrens Research Institute, Parkville, Victoria, Australia

${ }^{7}$ Deakin Health Economics, Deakin University, Burwood, Victoria, Australia

Acknowledgements The authors are grateful to all the trial sites for their support of the DAME trial. They also thank the women and their babies participating in this trial.

Contributors DAF, AMM and KM conceptualised the study, and SJ, LHA, PD, SPW, GO, SMD, RF, CM, AA and LG contributed to study design. DAF, LHA, AMM, KM drafted protocol and all authors contributed to protocol revision and application of grant. DAF, LHA, AMM developed data collection tools and all authors contributed to refinement, piloting and completion of tools. AMM, $\mathrm{KM}, \mathrm{LHA}, \mathrm{GO}$, SJ and DAF piloted and refined the intervention. AMM, DAF, AA and $\mathrm{CM}$ developed the recruitment processes. DAF, AMM and LHA developed the data collection process. DAF and LHA drafted the trial protocol manuscript, and all authors read and contributed to drafts, and read and approved final manuscript.

Funding This trial is funded by a project grant from the National Health and Medical Council of Australia (no. 1005345). We have also received equipment grants from the National Health and Medical Council of Australia.

Competing interests None.

Ethics approval La Trobe University and participating hospital sites.

Provenance and peer review Not commissioned; externally peer reviewed. 
Open Access This is an Open Access article distributed in accordance with the Creative Commons Attribution Non Commercial (CC BY-NC 4.0) license, which permits others to distribute, remix, adapt, build upon this work noncommercially, and license their derivative works on different terms, provided the original work is properly cited and the use is non-commercial. See: http:// creativecommons.org/licenses/by-nc/4.0/

\section{REFERENCES}

1. Begg SJ, Vos T, Barker B, et al. Burden of disease and injury in Australia in the new millennium: measuring health loss from diseases, injuries and risk factors. Med J Aust 2008;188:36-40.

2. American Diabetes Association. Diagnosis and classification of diabetes mellitus. Diabetes Care 2010;33(Suppl 1):S62-9.

3. Ratner RE, Christophi CA, Metzger BE, et al. Prevention of diabetes in women with a history of gestational diabetes: effects of metformin and lifestyle interventions. J Clin Endocrinol Metab 2008;93:4774-9.

4. Australian Institute of Health and Welfare. Diabetes prevalence in Australia: an assessment of national data sources. Canberra, 2009.

5. Mitanchez D, Burguet A, Simeoni U. Infants born to mothers with gestational diabetes mellitus: mild neonatal effects, a long-term threat to global health. $J$ Pediatr 2014;164:445-50.

6. Landon MB, Spong CY, Thom E, et al. A multicenter, randomized trial of treatment for mild gestational diabetes. N Engl J Med 2009;361:1339-48.

7. Jensen DM, Korsholm L, Ovesen P, et al. Peri-conceptional A1C and risk of serious adverse pregnancy outcome in 933 women with type 1 diabetes. Diabetes Care 2009;32:1046-8.

8. Garcia-Patterson A, Gich I, Amini SB, et al. Insulin requirements throughout pregnancy in women with type 1 diabetes mellitus: three changes of direction. Diabetologia 2010;53:446-51.

9. Metzger BE, Lowe LP, Dyer AR, et al; HAPO Study Cooperative Research Group. Hyperglycemia and adverse pregnancy outcomes. N Engl J Med 2008;358:1991-2002.

10. Mitanchez D. Foetal and neonatal complications in gestational diabetes: perinatal mortality, congenital malformations, macrosomia, shoulder dystocia, birth injuries, neonatal complications. Diabetes Metab 2010;36:617-27.

11. Cardwell CR, Stene LC, Ludvigsson J, et al. Breast-feeding and childhood-onset type 1 diabetes: a pooled analysis of individual participant data from 43 observational studies. Diabetes Care 2012;35:2215-25.

12. Patelarou E, Girvalaki C, Brokalaki H, et al. Current evidence on the associations of breastfeeding, infant formula, and cow's milk introduction with type 1 diabetes mellitus: a systematic review. Nutr Rev 2012;70:509-19.

13. Ip S, Chung M, Raman G, et al. Breastfeeding and maternal and infant health outcomes in developed countries. Rockville, MD: Agency for Healthcare Research and Quality, 2007.

14. Webster J, Moore K, McMullan A. Breastfeeding outcomes for women with insulin dependent diabetes. J Hum Lact 1995;11:195-200.

15. Hummel S, Winkler C, Schoen S, et al. Breastfeeding habits in families with Type 1 diabetes. Diabet Med 2007;24:671-6.

16. Schoen S, Sichert-Hellert W, Hummel S, et al. Breastfeeding duration in families with type 1 diabetes compared to non-affected families: results from BABYDIAB and DONALD studies in Germany. Breastfeed Med 2008;3:171-5.

17. Finkelstein SA, Keely E, Feig DS, et al. Breastfeeding in women with diabetes: lower rates despite greater rewards. A population-based study. Diabet Med 2013;30:1094-101.

18. Stage $E$, Norgard $H$, Damm $P$, et al. Long-term breast-feeding in women with type 1 diabetes. Diabetes Care 2006;29:771-4.

19. Kulski JK, Smith M, Hartmann PE. Perinatal concentrations of progesterone, lactose and alpha-lactalbumin in the mammary secretion of women. $J$ Endocrinol 1977;74:509-10.

20. Murtaugh MA, Ferris AM, Capacchione CM, et al. Energy intake and glycemia in lactating women with type 1 diabetes. J Am Diet Assoc 1998;98:642-8.

21. Arthur PG, Kent JC, Hartmann PE. Metabolites of lactose synthesis in milk from diabetic and nondiabetic women during lactogenesis II. $J$ Pediatr Gastroenterol Nutr 1994;19:100-8.

22. Bitman J, Hamosh M, Hamosh P, et al. Milk composition and volume during the onset of lactation in a diabetic mother. Am J Clin Nutr 1989:50:1364-9.

23. Miyake A, Tahara M, Koike K, et al. Decrease in neonatal suckled milk volume in diabetic women. Eur J Obstet Gynecol Reprod Biol 1989;33:49-53.

24. Neubauer SH, Ferris AM, Chase CG, et al. Delayed lactogenesis in women with insulin-dependent diabetes mellitus. Am J Clin Nutr 1993;58:54-60.
25. Nommsen-Rivers LA, Dolan LM, Huang B. Timing of stage II lactogenesis is predicted by antenatal metabolic health in a cohort of primiparas. Breastfeed Med 2012;7:43-9.

26. Hawkins JS, Casey BM. Labor and delivery management for women with diabetes. Obstet Gynecol Clin N Am 2007;34:323-34.

27. Beucher G, Viaris de Lesegno B, Dreyfus M. Maternal outcome of gestational diabetes mellitus. Diabetes Metab 2010;36:522-37.

28. Amir LH, Donath S. A systematic review of maternal obesity and breastfeeding intention, initiation and duration. BMC Pregnancy Childbirth 2007;7:9.

29. Schaefer-Graf UM, Hartmann R, Pawliczak J, et al. Association of breast-feeding and early childhood overweight in children from mothers with gestational diabetes mellitus. Diabetes Care 2006;29:1105-7.

30. Remsberg KE, McKeown RE, McFarland KF, et al. Diabetes in pregnancy and cesarean delivery. Diabetes Care 1999;22:1561-7.

31. Simmons D, Conroy C, Thompson CF. In-hospital breast feeding rates among women with gestational diabetes and pregestational Type 2 diabetes in South Auckland. Diabetic Med 2005;22:177-81.

32. Renfrew M, Craig D, Dyson L, et al. Breastfeeding promotion for infants in neonatal units: a systematic review and economic analysis. Health Technol Assess 2009;13:1-146.

33. Cox SG. Expressing and storing colostrum antenatally for use in the newborn period. Breastfeed Rev 2006;14:11-16.

34. Rietveld C. Antenatal colostrum harvesting for pregnant women with diabetes mellitus (DM) (Poster). Midwifery Conference. New Zealand, 2011.

35. Chapman T, Pincombe J, Harris M, et al. Antenatal breast expression: exploration and extent of teaching practices amongst International Board Certified Lactation Consultant midwives across Australia. Women Birth 2013;26:41-8.

36. Brisbane JM, Giglia RC. Experiences of expressing and storing colostrum antenatally: a qualitative study of mothers in regional Western Australia. J Child Health Care. Published Online First: 23 October 2013. doi 10.1177/1367493513503586

37. Tozier PK. Colostrum versus formula supplementation for glucose stabilization in newborns of diabetic mothers. JOGGN 2013;42:619-26.

38. East CE, Dolan WJ, Forster DA. Antenatal breast milk expression by women with diabetes for improving infant outcomes. Cochrane Database Syst Rev 2014;7:CD010408.

39. Cairns and Hinterland Health Service District. Antenatal expression of colostrum procedure. Queensland Government, 2008.

40. Logan Hospital, Women's and Children's Health Service. Breastfeeding Information for Pregnant Women with Diabetes. 2009

41. West D, Marasco L. Getting your milk supply off to a good start. The breastfeeding mother's guide to making more milk. New York: McGraw-Hill, 2009:57-67.

42. Soltani H, Scott AM. Antenatal breast expression in women with diabetes: outcomes from a retrospective cohort study. Int Breastfeed $J$ 2012;7:18.

43. Forster DA, McEgan K, Ford R, et al. Diabetes and antenatal milk expressing: a pilot project to inform the development of a randomised controlled trial. Midwifery 2011;27:209-14.

44. Forster DA, Amir LH, Moorhead A, et al. Is not testing a major change in clinical practice an ethical dilemma? (Letter). Midwifery 2011;27:e301-2.

45. Lawrence RA, Lawrence RM. Diabetes mellitus. Breastfeeding: a guide for the medical profession. 5th edn. St Louis: Mosby, 1999:515-21.

46. Ho HT, Yeung WK, Young BW. Evaluation of "point of care" devices in the measurement of low blood glucose in neonatal practice. Arch Dis Child Fetal Neonatal Ed 2004;89:F356-9.

47. Bonacruz GL, Arnold JD, Leslie Gl, et al. Survey of the definition and screening of neonatal hypoglycaemia in Australia. $J$ Paediatr Child Health 1996;32:299-301.

48. Australian Government Department of Health and Ageing. National hospital cost data collection, cost weights for AR-DRG version 6.0x, round 14 (2009-10). Canberra, 2012.

49. Australian Government Department of Health and Ageing. Medical benefits schedule book. Canberra, 2011.

50. Schulz KF, Altman DG, Moher D. CONSORT 2010 statement: updated guidelines for reporting parallel group randomised trials. PLOS Med 2010;7:e1000251.

51. Ezzy D. Coding data and interpreting text: methods of analysis Qualitative analysis: practice and innovation. Sydney: Allen \& Unwin 2002:81-110

52. Cox S. An ethical dilemma: should recommending antenatal expressing and storing of colostrum continue? Breastfeed Rev 2010;18:5-7

53. Chapman T, Pincombe J, Harris M. Antenatal breast expression: a critical review of the literature. Midwifery 2013;29:203-10. 